\title{
Twelve-Year Single Center Experience Shows Safe Implementation of Developed Peritoneal Surface Malignancy Treatment Protocols for Gastrointestinal and Gynecological Primary Tumors
}

\author{
Philipp Horvath ${ }^{1}$, Can Yurttas ${ }^{1}\left(\mathbb{D}\right.$, Stefan Beckert ${ }^{1,2}$, Alfred Königsrainer ${ }^{1}$ and Ingmar Königsrainer ${ }^{1,3, *}$ \\ 1 Comprehensive Cancer Center, Department of General, Visceral and Transplant Surgery, \\ University of Tübingen, 72076 Tübingen, Germany; philipp.horvath@med.uni-tuebingen.de (P.H.); \\ can.yurttas@med.uni-tuebingen.de (C.Y.); Stefan.Beckert@sbk-vs.de (S.B.); \\ alfred.koenigsrainer@med.uni-tuebingen.de (A.K.) \\ 2 Department of General and Visceral Surgery, Schwarzwald-Baar Klinikum, \\ 78052 Villingen-Schwenningen, Germany \\ 3 Department of General, Visceral and Thoracic Surgery, Academic Teaching Hospital Feldkirch, \\ Carinagasse 47, 6800 Feldkirch, Austria \\ * Correspondence: ingmar.koenigsrainer@lkhf.at
}

Citation: Horvath, P.; Yurttas, C.; Beckert, S.; Königsrainer, A.; Königsrainer, I. Twelve-Year Single Center Experience Shows Safe Implementation of Developed Peritoneal Surface Malignancy Treatment Protocols for Gastrointestinal and Gynecological Primary Tumors. Cancers 2021, 13, 2471. https://doi.org/10.3390/ cancers13102471

Academic Editor: Kuno Lehmann

Received: 26 March 2021

Accepted: 11 May 2021

Published: 19 May 2021

Publisher's Note: MDPI stays neutral with regard to jurisdictional claims in published maps and institutional affiliations.

Copyright: (c) 2021 by the authors. Licensee MDPI, Basel, Switzerland. This article is an open access article distributed under the terms and conditions of the Creative Commons Attribution (CC BY) license (https:// creativecommons.org/licenses/by/ $4.0 /)$.
Simple Summary: The treatment of peritoneal surface malignancies (PSM) has dramatically evolved during the past two decades. Indications, treatment protocols, surgical techniques and the application of HIPEC in the prophylactic setting were evaluated in the surgical community. Nevertheless, the current results of the PRODIGE-7 trial disfavored the application of HIPEC for PSM of colorectal cancer and raised uncertainty among surgeons. On the other hand, cytoreductive surgery and HIPEC represent state-of-the-art therapy for peritoneal mesothelioma (except the sarcomatoid-subtype) and pseudomyxoma peritonei. Comparing the literature is cumbersome due to the variety of HIPEC protocols and differences in indication settings. This article aims to provide an insight into the impact of different HIPEC protocols, different indication settings and the implementation of pre-HIPEC laparoscopy on patients' morbidity rates and outcomes and serves as guidance for surgeons dealing with these patients in order to guarantee high-quality treatment.

Abstract: (1) Background: Cytoreductive surgery and hyperthermic intraperitoneal chemotherapy provide survival benefits to selected patients. We aimed to report our experience and the evolution of our peritoneal surface malignancy program. (2) Methods: From June 2005 to June 2017, 399 patients who underwent cytoreductive surgery plus hyperthermic intraperitoneal chemotherapy at the Tübingen University Hospital were analyzed from a prospectively collected database. (3) Results: Peritoneal metastasis from colorectal cancer was the leading indication (group 1: 28\%; group 2: $32 \%$ ). The median PCI was 15.5 (range, $1-39$ ) in group 1 and 11 (range, $1-39$ ) in group $2(p=0.002$ ). Regarding the completeness of cytoreduction (CC), a score of 0 was achieved in $63 \%$ vs. $69 \%$ for group 1 and 2 , respectively $(p=0.010)$. Median overall survival rates for patients in group 1 and 2 for colon cancer, ovarian cancer, gastric cancer and appendix cancer were 34 and 25 months; 45 months and not reached; 30 and 16 months; 39 months and not reached, respectively. The occurrence of grade-III and -IV complications slightly differed between groups (14.5\% vs. $15.6 \%)$. No 30-day mortality occurred. (4) Conclusions: Specialized centers are able to provide low-morbidity cytoreductive surgery and hyperthermic intraperitoneal chemotherapy without mortality. Strict patient selection during the time period significantly improved CC scores.

Keywords: peritoneal metastases; morbidity; outcome; HIPEC; cytoreductive surgery 


\section{Introduction}

The management of peritoneal surface malignancies (PSM) has experienced a crucial change in recent years. Once an inoperable and palliative situation, with the only therapeutic option being systemic chemotherapy, there is now a curative treatment that can be provided for a selected patient subset. The acclaim of hyperthermic intraperitoneal chemotherapy (HIPEC) subsided after results of the PRODIGE-7-, PRODIGE 15and COLOPEC-trial [1-3] were presented. The high-dose and short-term oxaplatin (OX)based HIPEC is now widely abandoned, and other HIPEC compounds, such as cisplatin and mitomycin C (MMC), have regained attention. Above all, the PRODIGE-7 trial was criticized due to a variety of methodological weaknesses, including an expected overoptimistic survival in the experimental arm, combined with an underestimation of the effect of cytoreductive surgery alone [4]. Furthermore, the short drug exposure time of OX, the possible adverse effects of the carrier solution (Dextrose $5 \%$ ) and possible adverse effects of hyperthermia itself were considered, explaining the negative results of the study. The same is true for the PRODIGE-15 study, which was mainly criticized due to a highly heterogenous study population (non-metastatic and metastatic setting) [5].

To date, no randomized controlled trial comparing the efficacy of different HIPEC compounds exists, but a recent meta-analysis, including 11 studies and 2091 patients, comparing MMC- and OX-based HIPEC, suggested no evidence for differences in overall and disease-free survival but a statistically significantly increased rate of major complications to the disadvantage of OX [6].

Undoubtedly, the value of a high-quality cytoreductive surgery (CRS) is still a crucial cornerstone in order to provide satisfactory survival rates for patients with PSM. The PRODIGE-7 trial, despite the lack of efficacy of additional HIPEC, showed a median overall survival rate of 41 months, bearing in mind that approximately $25 \%$ of patients had PCI scores (peritoneal cancer index) $>16$, and patients with signet-ring histology were also included. During the recruitment periods of the abovementioned randomized trials, indications in favor of CRS and HIPEC, for almost all tumor etiologies, narrowed. On the other hand, evidence grew, that concomitant colorectal liver and peritoneal metastases should not contraindicate combined surgery and HIPEC for a strictly selected patient subset, here defined by their response to systemic chemotherapy, a low to moderate PCI $(<17)$ and a maximum of three good resectable liver metastases (LM) [7]. Apart from the PCI score, a variety of tumor-, patient- and treatment-specific parameters now impact the treatment algorithm. A favorable PCI (colorectal: <16 [8]; gastric: <6-10 [9]; ovarian: not defined yet [10-12]), a favorable histology (signet-ring histology is considered a relative contraindication for CRS and HIPEC $[13,14]$ ) and response to systemic chemotherapy should be present. These parameters should allow for a proper patient selection and, in combination with a structured perioperative complication management, acceptable morbidity and mortality rates should be achievable. Recent data have shown that, over time, a lower percentage of patients died due to manageable but potentially life-threatening complications after CRS and HIPEC, suggesting a higher expertise in patient selection and complication management [15]. Furthermore, data showed that cumulative in-hospital mortality after CRS and HIPEC over a 9-year period was only 3.4\% in Germany, which is lower than for oncologic pancreas, esophagus and liver surgery [15]. Center experience is another factor impacting on postoperative morbidity [16] after CRS and HIPEC, emphasizing the importance of high-volume centers.

This whole evolution process of PSM treatment has impacted on many centers of excellence in terms of HIPEC compounds, indications and perioperative management. We, therefore, aimed to depict this process at our institution and report our experience on different HIPEC drugs, means of application, indications and postoperative outcomes for patients undergoing CRS and HIPEC for gastrointestinal and gynecological primary tumors. 


\section{Materials and Methods}

\subsection{Patient Selection Criteria}

From June 2005 to June 2017, a total of 399 patients underwent CRS and HIPEC for PSM of various gastrointestinal and gynecological cancers and were considered for this retrospective analysis. The patients were divided into two subgroups (group 1: 2005-2012 and group 2: 2013-2017). The main differences were the chemotherapy regimen, the application procedure (open-HIPEC and only i.p. application in group 1 and closed-HIPEC and i.v/i.p. application in group 2), indication setting and introduction of diagnostic laparoscopy pre-CRS/HIPEC.

Clinicopathological information was obtained from a prospectively collected database and electronic medical reports. The study was performed according to the guidelines of the local institutional board and the ethics committee (610/2017BO2).

Preoperative diagnostics consisted of thorough clinical examination, blood tests and a computed tomography (CT) scan. CT images were acquired with a 128-slice multidetector spiral CT. The reconstructed slice thickness was $5 \mathrm{~mm}$ without gaps between slices. Irresectability for CRS and HIPEC was defined as infiltration of the mesenteric axis, retroperitoneal plane or the pancreatic head. Irresectability regarding LM was dictated by metastases located in both liver lobes that were not suitable for atypical resection and that needed extended liver resection. Eligibility for CRS and HIPEC and resection of concurrent liver metastasis was assessed by a surgical oncologist, a medical oncologist, a radiologist and a radio-oncologist, all of whom attended the interdisciplinary oncologic team meeting. Adverse events were classified according to the Clavien-Dindo complication score [17].

\subsection{Indications}

Compared to group 1, patients in group 2 were only considered for CRS and HIPEC if they met the following parameters:

- Deemed cytoreducable on laparoscopy;

- Response to systemic chemotherapy;

- PCI cut-offs (gastric: 6-10; colorectal/appendix <16; Mesothelioma/PMP/Ovarian: no cut-off).

Patients with signet-ring histology or RAS/BRAF mutations were not excluded from CRS and HIPEC in both patient groups.

\subsection{Cytoreductive Surgery}

After laparotomy through a mid-line incision and complete adhesiolysis, the PCI was determined following the criteria described by Jaquet and Sugarbaker [16]. Abdominal regions were categorized as the small bowel, consisting of Sugarbaker's abdominopelvic regions (SAPR) 9 to 12; the upper abdomen, consisting of SAPR 0 to 3; and the lower abdomen/pelvis consisting of SAPR 4 to 8 . Tumor-involved structures were resected along with peritonectomy procedures described by Sugarbaker [18-20] aiming for complete cytoreduction (CC-0 and CC-1 (CC-0 indicated no visible disease; CC-1 indicated nodules smaller than $0.25 \mathrm{~cm}$; CC-2 indicated nodules greater than $0.25 \mathrm{~cm}$ and smaller than $5 \mathrm{~cm}$; CC-3 indicated nodules over $5 \mathrm{~cm})$ ).

\subsection{HIPEC}

After complete cytoreduction and fashioning of intestinal anastomoses, HIPEC was administered for 30 to $90 \mathrm{~min}$ at $42{ }^{\circ} \mathrm{C}$, depending on the HIPEC compound, using the open- (group 1) or the closed abdomen (group 2) technique. The dosage for oxaliplatin was $300 \mathrm{mg} / \mathrm{m}^{2}$, for mitomycin C was $35 \mathrm{mg} / \mathrm{m}^{2}$ and for cisplatin was $75 \mathrm{mg} / \mathrm{m}^{2}$ body surface area. Patients receiving oxaliplatin i.p. $\left(300 \mathrm{mg} / \mathrm{m}^{2} ; 30 \mathrm{~min}\right.$ ) also simultaneously received 5-FU $\left(400 \mathrm{mg} / \mathrm{m}^{2}\right)$ i.v. In group 1, MMC-based $(90 \mathrm{~min})$ or cisplatin-based (60 $\left.\mathrm{min}\right)$ HIPEC was administered, whereas in group 2, a bi-directional chemotherapy protocol (OX (i.p.)/5-FU (i.v.) or a combination of cisplatin/doxorubicin for HIPEC was administered. 
After HIPEC completion, the abdomen was washed out with $3 \mathrm{~L}$ of Ringer's lactate solution and the abdomen was reopened for the removal of the perfusion catheters before the fascial closure was performed.

\subsection{Statistics}

Stata SE 13 was used for survival analysis. All analyses were stratified by group 1 and 2. Subgroups examined were $\mathrm{PCI}<17$ and $\mathrm{PCI} \geq 17$; G2/G3; N0/N1/N2; CC0/CC1; WT and MUT. After descriptive analysis of the data (i.e., examining survival time, incidence rate and the 25, 50 and $75 \%$ survival time), log rank tests were used to examine whether the survival functions were equal across the groups. Lastly, Kaplan-Meier survival curves were used to visualize survival across the subgroups.

\section{Results}

From June 2005 to June 2017, a total of 399 patients underwent CRS and HIPEC. Group 1 contained 237 patients, while group 2 contained 162. The median age did not differ between the groups ( 55.3 vs. 54.2 years; $p=0.30$ ). Furthermore, sex distribution was also similar ( $34.2 \%$ males vs. $42 \%$ males; $p=0.070)$. In both groups, peritoneal metastasis (PM) mainly originated from colorectal cancer (CRC) (28\% vs. $32 \%)$. The proportion of patients with recurrent PM of ovarian origin significantly decreased when comparing group 1 and group 2 , respectively $(27 \%$ vs. $6 \% ; p<0.0001)$. The remaining tumor etiologies are listed in Table 1.

Table 1. Patient- and treatment-related parameters (5-FU $=5$-Fluorouracil; $\mathrm{CC}=$ completeness of cytoreduction; $\mathrm{CRC}=$ colorectal cancer; i.p. = intraperitoneal; i.v. = intravenous; $\min =$ minutes; $\mathrm{MMC}=$ mitomycin $\mathrm{C} ; \mathrm{OX}=$ oxaliplatin; $\mathrm{PCI}=$ peritoneal cancer index; PMP = pseudomyxoma peritonei; SSI = surgical site infections).

\begin{tabular}{|c|c|c|c|}
\hline Parameter & Group $1(n=237)$ & Group $2(n=162)$ & $p$-Value \\
\hline Median Age (range) & $55.3(14-75)$ & $54.2(19-79)$ & 0.3 \\
\hline Sex (male) $\%(n)$ & $34(81)$ & $42(68)$ & 0.07 \\
\hline \multicolumn{4}{|l|}{ Tumor etiology $\%(n)$} \\
\hline CRC & $28(67)$ & $32(51)$ & 0.268 \\
\hline Ovarian & $27(64)$ & $6(10)$ & $<0.0001$ \\
\hline Gastric & $12(28)$ & $12(19)$ & 0.979 \\
\hline Appendix & $9(21)$ & $17(28)$ & 0.011 \\
\hline Mesothelioma & $5(12)$ & $3(5)$ & 0.337 \\
\hline PMP & $11(26)$ & $17(28)$ & 0.075 \\
\hline Others & $8(19)$ & $13(21)$ & 0.648 \\
\hline Median PCI (range) & $15.5(1-39)$ & $11(1-39)$ & 0.002 \\
\hline Operative times (min) & $541(107-1076)$ & $315.5(66-770)$ & $<0.001$ \\
\hline \multicolumn{4}{|l|}{ CC-score \% (n) } \\
\hline CC -0 & $63(150)$ & 69 (112) & \\
\hline CC-1 & $30(71)$ & $31(50)$ & 0.010 \\
\hline CC-2 & $4(9)$ & - & \\
\hline CC-3 & $3(7)$ & - & \\
\hline HIPEC technique & open & closed & \\
\hline \multicolumn{4}{|l|}{ HIPEC compound \% ( $n)$} \\
\hline $\mathrm{MMC}$ & $54(128)$ & - & \\
\hline Cisplatin & $41(97)$ & - & \\
\hline MMC/Cisplatin & $3(8)$ & - & \\
\hline OX (i.p.)/5-FU (i.v.) & - & $61(98)$ & \\
\hline OX & - & $3(6)$ & \\
\hline Cisplatin/Doxorubicin & - & $30(48)$ & \\
\hline Others & $2(4)$ & $6(10)$ & \\
\hline
\end{tabular}


Table 1. Cont.

\begin{tabular}{|c|c|c|c|}
\hline Parameter & Group $1(n=237)$ & Group $2(n=162)$ & $p$-Value \\
\hline \multicolumn{4}{|l|}{ HIPEC duration } \\
\hline Cisplatin-based & $60 \mathrm{~min}$ & $30 \mathrm{~min}$ & \\
\hline MMC-based & $90 \mathrm{~min}$ & & \\
\hline \multicolumn{4}{|l|}{ Resections \% (n) } \\
\hline Omentectomy & 49 (116) & $64(104)$ & 0.003 \\
\hline Appendectomy & $11(26)$ & $20(32)$ & 0.339 \\
\hline Splenectomy & $31(73)$ & $7(11)$ & $<0.0001$ \\
\hline Rectum & $25(59)$ & $12(19)$ & 0.007 \\
\hline Small bowel & $23(55)$ & $13(21)$ & 0.014 \\
\hline Internal genitals & $23(55)$ & $20(32)$ & 0.323 \\
\hline Right colon & $17(40)$ & $14(23)$ & 0.078 \\
\hline Gastric & $17(40)$ & $19(31)$ & 0.562 \\
\hline \multicolumn{4}{|l|}{ Complication rate $\%(n)$} \\
\hline Total & $60(142)$ & $62(101)$ & 0.21 \\
\hline > Grade IIIa & $16(37)$ & $13(21)$ & 0.461 \\
\hline \multicolumn{4}{|l|}{ Complication type \% (n) } \\
\hline Leucopenia & $34(81)$ & $16(26)$ & $<0.0001$ \\
\hline $\begin{array}{l}\text { Anastomotic } \\
\text { insufficiency }\end{array}$ & $4(9)$ & $6(10)$ & 0.332 \\
\hline Pleural effusion & $10(24)$ & $23(37)$ & 0.091 \\
\hline Pneumonia & $4(9)$ & $3(5)$ & 0.704 \\
\hline Pulmonary embolism & $6(14)$ & $3(5)$ & 0.152 \\
\hline Fascial rupture & $3(7)$ & $6(10)$ & 0.588 \\
\hline SSI & $10(24)$ & $6(10)$ & 0.079 \\
\hline Back to theatre & $15(36)$ & $12(20)$ & 0.421 \\
\hline 30-day mortality & $0 \%$ & $0 \%$ & \\
\hline Hospital stay (days (range)) & $17(3-105)$ & $14(6-74)$ & $<0.0001$ \\
\hline
\end{tabular}

In group 1, 54\% received MMC-based and 41\% cisplatin-based HIPEC, whereas in group 2, 61\% received a bi-directional chemotherapy protocol (OX (i.p.)/5-FU (i.v.) and $30 \%$ received a combination of cisplatin/doxorubicin for HIPEC. The median PCI for all patients was 14, with a significant decrease comparing group 1 and 2 (15.5 vs. $11 ; p=0.002$ ). Operative time also decreased significantly over time (541 vs. $315 \mathrm{~min} ; p<0.0001)$. In group 1, in 93\% of patients, a CC-0/1 resection was achieved (CC-0: 63\%, CC-1: 30\%). In group 2, in 100\% of patients, a CC-0/1 status was attained (CC-0: 69\%; CC-1: 31\%) $(p=0.010)$. During the same period, 165 patients received explorative laparotomy or debulking surgery only. The number of patients treated without HIPEC declined from group 1 to group 2 (105 vs. 65 patients; $p=0.137$ ).

For the achievement of a CC- $0 / 1$ score, a variety of visceral resections were necessary (Table 1). In both groups, omentectomy was carried out the most frequently (49\% vs. 64\%).

\subsection{Morbidity and Mortality}

The total complication rate was comparable between both groups (60\% vs. 62\%; $p=0.210)$. The rate of complications equal to or larger than grade III was also similar in both groups (16\% vs. 13\%). Re-operation was necessary in $12 \%$ of group 1 and in $15 \%$ of group 2 and was also comparable. The most common complication in group 1 was postoperative leucopenia (34 \% vs. $16 \% ; p<0.0001$ ). In group 2 , the most common complication was the occurrence of pleural effusion (10\% vs. $23 \% ; p=0.091)$. Instances of anastomotic insufficiencies $(4 \%$ vs. $6 \% ; p=0.332)$ and fascial rupture $(3 \%$ vs. $6 \% ; p=0.588)$ were low and comparable between both groups. Further complications are listed in Table 1. In both groups, no in-hospital or 30-day mortality occurred. The length of hospital stay differed significantly between both groups (17 vs. 14 days; $p<0.0001$ ). 


\subsection{Survival Data}

The median overall survival for patients in group 1 and 2 for colon cancer, ovarian cancer gastric cancer and appendix cancer were 34 (range, 1-85) and 25 (range, 3-42) months; 45 (range, 10-142) months and not reached; 30 (range, 9-117) and 16 (range, 5-32) months) and 39 (range, 32-61) months and not reached (Table 2, Figures 1 and 2), respectively.

Table 2. Median overall survival for each tumor etiology $(C R C=$ colorectal cancer; $O S=$ overall survival; n.r. $=$ not reached)

\begin{tabular}{cc}
\hline Tumor Etiology & Median OS (Months (Range)) \\
\hline CRC & Group 1: 34 (1-85) \\
Ovarian & Group 2: 25 (3-42) \\
& Group 1: $45(10-142)$ \\
Gastric & Group 2: n.r. \\
& Group 1: 30 $(9-117)$ \\
Appendix & Group 2: 16 (5-32) \\
& Group 1: 39 (32-61) \\
\hline
\end{tabular}

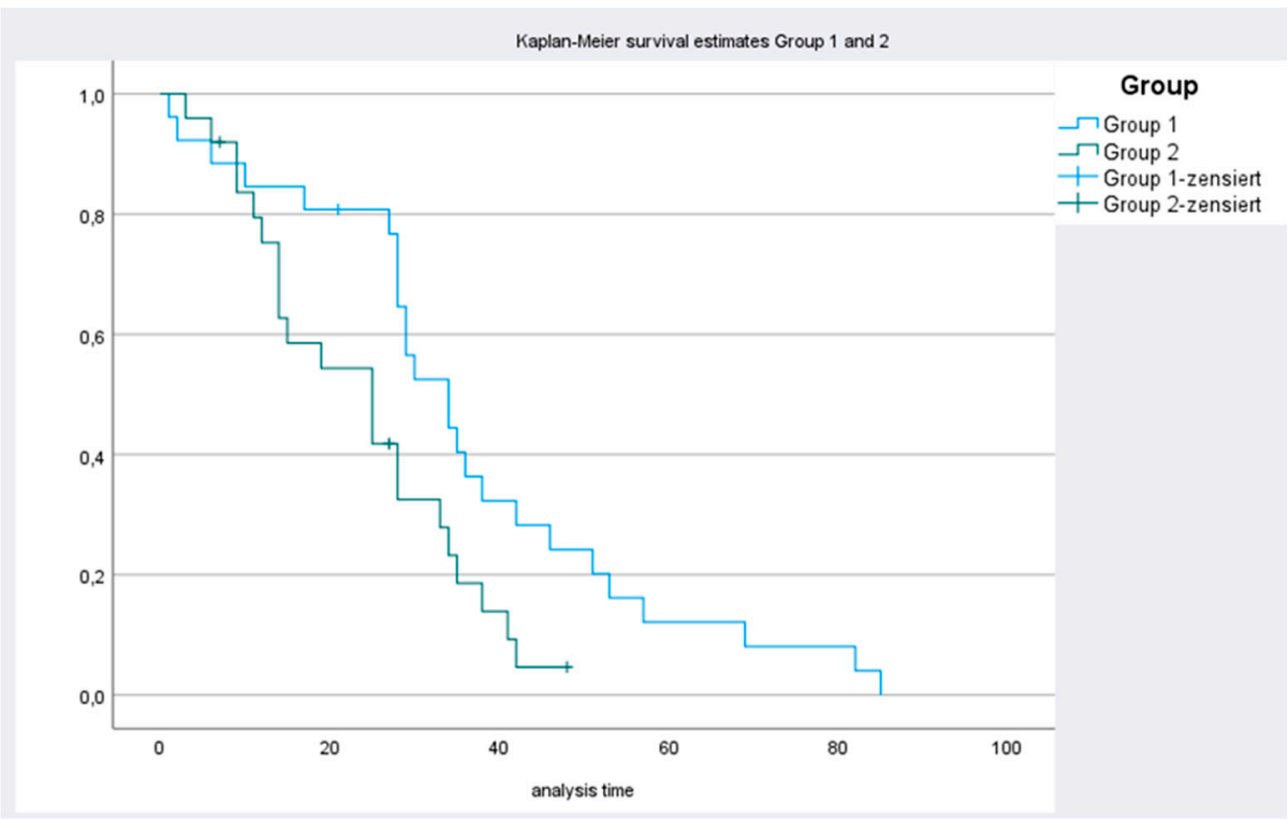

Figure 1. Kaplan-Meier curves for patients with CRC in group 1 and 2.

Patients with CRC in group 2 showed a trend towards a better overall survival with low to intermediate PCI values $(<17)$, but did not reach statistical significance ( $p=0.183$ ). Furthermore, in CRC for both groups, the CC score (CC-0 vs. CC- 1 ), grading (G2 vs. G3) and lymphonodal status (N0 vs. N1 vs. N2) did not significantly impact overall survival, respectively. 


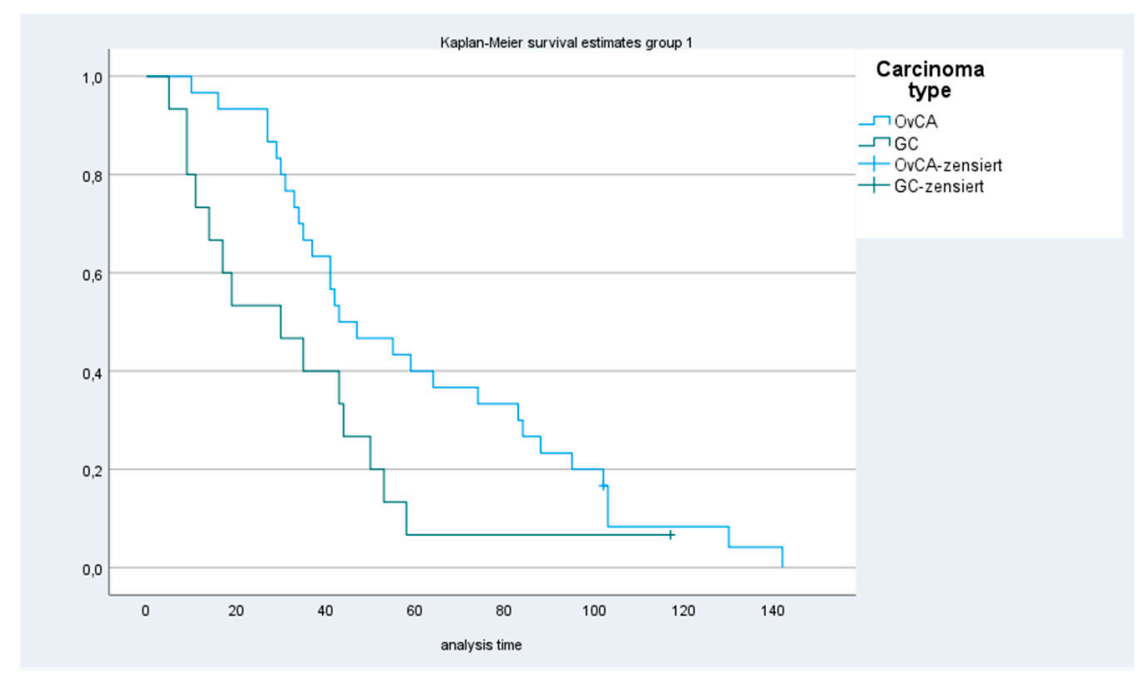

Figure 2. Kaplan-Meier curves for patients with ovarian cancer (OvCA) and with gastric cancer (GC).

\section{Discussion}

The implementation of additional flushing of the abdominal cavity with a hyperthermic chemotherapeutic solution after maximal cytoreductive surgery was the milestone in the surgical treatment of peritoneal surface malignancies. Since the first description by Spratt and co-workers in the early 1980s, a variety of experimental and clinical research has been undertaken in order to generate parameters helping surgeons to perform a strict patient selection and to provide a safe procedure to patients who are likely to benefit the most from this aggressive surgical approach [19].

Our current analysis aimed to investigate the impact of different HIPEC protocols and indication setting on long-term results, especially focusing on differing PCI and CC scores and postoperative morbidity and mortality. Strict patient selection is imperative in order to provide a low-morbidity and low-mortality procedure. This means that only patients with favorable tumor characteristics and a low tumor burden should be included in order to minimize the risk of non-therapeutic laparotomies.

A retrospective cohort, multicentric study from 23 French centers showed that the $\mathrm{PCI}$ and the experience of the center were statistically significantly linked to increased postoperative morbidity. Centers were classified as experienced ( $>7$ years of practice) and as inexperienced ( $<7$ years of practice). These data once again highlight the importance of center experience in order to provide low-morbidity CRS and HIPEC [16]. Recently, health insurance data from Germany showed that over a 9-year period, the outcome of manageable but life-threatening complications after CRS and HIPEC significantly improved, resulting in a cumulative in-hospital mortality of 3.4\% [15]. Data show that at least 141 procedures have to be performed to gain sufficient expertise [20]. In the study from Huang et al., 800 patients who received CRS and HIPEC were divided into two equal groups and compared [21]. The authors stated that patients in the second group had a significantly lower PCI, a better resection status and lower morbidity and mortality rates. These results are in line with ours, which also show that patients in group 2 had a significantly lower PCI, and a significantly higher proportion of patients received a complete resection (CC-0/1). The phenomenon of a decreasing PCI over time is attributable to a better patient selection, which is expressed by our declining rate of non-therapeutic laparotomies which can partially be attributed to the implementation of pre-HIPEC laparoscopy. Hentzen et al. stated that, after implementation of routinely performed laparoscopies prior to CRS and HIPEC, a significant decrease in non-therapeutic laparotomies was experienced ( $21 \%$ vs. $35.4 \% ; p=0.044)$ [22]. Likewise, Iversen and co-workers showed that laparoscopy is a useful tool in the patient selection process, but only 17 out of 27 patients, who were deemed amenable to CRS and HIPEC by laparoscopy, were classified as resectable on laparotomy [23]. This is in line with our experience; the PCI evaluated by laparoscopy is approximately $30 \%$ too low. 
In our patient cohort, the median PCI significantly decreased from 15.5 to 11 over time, which resulted in a significantly higher proportion of CC-0 resections. One of the most consistent independent predictors of morbidity is the extent of disease, measured by the PCI [24-28]. In our study, we also made the observation that a lower PCI was linked to fewer grade-III complications (15.6\% vs. $13 \%)$. A higher PCI is linked to a more aggressive surgery, thus triggering postoperative complications. Saxena et al. analyzed 145 patients receiving CRS and HIPEC for PMP and showed that a PCI $>21$ and an ASA score $>3$ were linked to grade-IV/V morbidity [29]. Furthermore, the literature suggests that the independent contribution of HIPEC to morbidity seems to be quite low. Yang et al. compared patients with CRS only and CRS plus HIPEC in patients with PM originating from gastric cancer and found no significant difference in the occurrence of serious adverse events $(11.7 \%$ vs. $14.7 \% ; p=0.839)$ [30]. The same results were reported by Bonnot et al., comparing CRS alone and CRS plus HIPEC for PM of gastric cancer, showing a similar major complication rate in both groups $(53.7 \%$ vs. $55.3 \% ; p=0.496)$ [31].

For CRS and HIPEC, it is almost impossible to attribute a complication exclusively to CRS or HIPEC. In our study, we observed a HIPEC-associated morbidity which was linked to the HIPEC compound used. As in many other publications, and over time, many different chemotherapeutic agents were used with different dosages and different perfusion times, making it impossible to compare data. In our study, we found that MMC-based HIPEC is a trigger for postoperative leucopenia. In total, $50.8 \%$ of patients who received MMC-based HIPEC developed leucopenia. This is in line with other reports [32,33]. In a former study of our group, the usage of either MMC or platin compounds was not linked to an increased morbidity. In this study, CRS and HIPEC were combined with liver resection, and the exposure of the liver resection margins to intraperitoneal heated chemotherapeutic agents did not trigger bile leakage or bleeding $[7,34]$.

In Germany, approximately 55\% of HIPEC procedures were performed for CRC-PM patients in 2018 [15], and the literature provides 5-year survival rates of up to $54 \%$ in patients treated with CRS and HIPEC [35]. The impact of systemic chemotherapy on CRC-PM was evaluated by a large database analysis. Franko and co-workers reviewed 10,635 patients with metastasized CRC, but only $1.9 \%(n=194)$ had CRC-PM, with a median overall survival of 16.3 months (with cytostatic agents) and 17.1 (with at least one targeted therapy) [36]. These data reveal two key facts: patients with isolated CRC-PM are highly outnumbered in clinical trials, and the effect of targeted therapy seems to be negligible. Compared to these results, the median OS of our patients was 34 and 25 months, respectively. To date, our survival rates for CRC-PM have not been reached by systemic chemotherapy. The decrease in survival in group 2 might be attributed to a high percentage of KRAS- and BRAF-mutated primary tumors compared to group 1 (12\% vs. $44 \%)$.

Recently, Schneider and co-workers were the first to show that KRAS (Hazard Ratio (HR) 1.46) and BRAF (HR 3.97) mutations of the primary tumor negatively affected survival after CRS and HIPEC [37]. Another recently published study found KRAS mutations (HR 2.02) to be an independent predictor of reduced OS after CRS and HIPEC [38] and Morgan and co-workers found KRAS mutations to be associated with early recurrence after CRS and HIPEC [39]. These data suggest that mutations in genomic driver genes impact on OS after CRS and HIPEC, but underlying studies were not randomized trials and mostly included a low number of patients. Furthermore, recent studies have revealed that BRAF mutations are associated with reduced OS, but no information is provided on the proportion of peritoneal and distant recurrent disease [40,41]. For the present time, evidence is too scarce to refuse these patients CRS and HIPEC in the event of detected mutations, because the true rate of peritoneal recurrent disease in KRAS- and BRAFmutated patients undergoing CRS and HIPEC is not known. These patients should rather be evaluated for an aggressive perioperative systemic chemotherapy in the context of CRS and HIPEC.

Recently, a German database analysis of 235 patients with PM of gastric cancer showed a median OS time of 13 months with a 5-year survival rate of $6 \%$. The median OS differed 
significantly according to the PCI range (0-6: 18 months; 7-15: 12 months; 16-39: 5 months; $p=0.002$ [ [42]. These data are line with ours, showing a median OS of 30 and 16 months for group 1 and 2, respectively. Likewise, the CYTO-CHIP study, comparing CRS alone and CRS and HIPEC for PM of gastric cancer, showed a prolongation of OS and recurrence-free survival (RFS) [31]. These analyses and our data outline the impact of immediate adjuvant HIPEC in the context of a very low peritoneal tumor burden on patient survival.

One main issue which was also discovered by our study was the significant decline in patients with ovarian cancer and PM. In the first group, the proportion was $27 \%$, and in the second group, $6 \%$. This phenomenon can be explained by the launch of the new German S3-guidelines for the therapy of ovarian cancer, which clearly disfavors the application of HIPEC. Despite the fact that reliable data on the effectiveness of additional HIPEC in ovarian cancer are available, they are still not included in the current treatment algorithm [7,43-45]. Of particular note are the results from Van Driel and colleagues, who compared interval CRS alone \pm HIPEC and showed a significant improvement in median recurrence-free survival (10.7 vs. 14.2 months; $p=0.003$ ) and median OS (33.9 vs. 45.7 months; $p=0.02$ ). The fact that only $67 \%$ and $69 \%$ of patients received macroscopic complete resection [45] is also noteworthy.

Contradictory results were provided by Coleman et al. reporting a significant difference in median OS between surgery plus systemic chemotherapy versus systemic chemotherapy alone in patients with platin-sensitive recurrent ovarian cancer, in favor of systemic chemotherapy alone [46]. The data should be interpreted with caution, because $10 \%$ of the patients had additional intra- or extra-abdominal metastasis, the PCI was not provided and a highly selected patient subset (platin-free interval of 20.4 and 18.8 months) was included.

Furthermore, in a previous study of our group, patients with peritoneal and hepatic metastases of ovarian cancer were subjected to CRS, HIPEC and liver resection, and in all patients, a radical macroscopic resection was achieved with a median overall survival of 30 months. Grade-III/IV morbidity rates were $23 \%$. These data show that even patients with ovarian cancer and PM can benefit from an aggressive surgical approach [7].

Due to the amount of clinical data favoring CRS and HIPEC for both primary and recurrent ovarian cancer, it seems increasingly incomprehensible that these patients are still refused CRS and HIPEC [47-50]. Furthermore, the ongoing criticism from gynecologists, namely, that CRS and HIPEC is a high-risk procedure, associated with high postoperative morbidity and mortality, thus delaying adjuvant systemic chemotherapy, must be invalidated, because recent data show the opposite [15,45]. The upcoming German guideline commission should, therefore, urgently redefine the clinical importance of CRS and HIPEC in ovarian cancer.

\section{Conclusions}

Strict patient selection and optimal perioperative management are crucial in order to provide low-morbidity and low-mortality CRS and HIPEC in selected patients with PM. Knowledge of patient- and treatment-related factors triggering postoperative morbidity is essential. In the near future, due to the overwhelming data favoring CRS and HIPEC for $\mathrm{PM}$ of ovarian cancer, selected patients should be urgently evaluated for this approach in order to further prolong OS and recurrence-free survival.

Author Contributions: Conceptualization, A.K. and I.K.; methodology, I.K. and P.H.; software, I.K.; validation, all; formal analysis, P.H., I.K. and C.Y.; investigation, P.H.; resources, I.K.; data curation, I.K.; writing-original draft preparation, I.K. and P.H.; writing-review and editing, S.B., A.K. and I.K.; visualization, S.B.; supervision, I.K.; project administration, A.K. All authors have read and agreed to the published version of the manuscript.

Funding: This research received no external funding.

Institutional Review Board Statement: The study was performed according to the guidelines of the local institutional board and the ethics committee (610/2017BO2). 
Informed Consent Statement: Informed consent was obtained from all subjects involved in the study.

Data Availability Statement: Data supporting results are harbored by an in-hospital database. Regulatory issues do not allow the provision of a link to analyzed data sets.

Conflicts of Interest: The authors declare no conflict of interest.

\section{References}

1. Quénet, F.; Elias, D.; Roca, L.; Goéré, D.; Ghouti, L.; Pocard, M.; Facy, O.; Arvieux, C.; Lorimier, G.; Pezet, D.; et al. Cytoreductive surgery plus hyperthermic intraperitoneal chemotherapy versus cytoreductive surgery alone for colorectal peritoneal metastases (PRODIGE 7): A multicentre, randomised, open-label, phase 3 trial. Lancet Oncol. 2021, 22, 256-266. [CrossRef]

2. Goere, D.; Glehen, O.; Quenet, F.; Guilloit, J.M.; Bereder, J.M.; Lorimier, G. BIG-RENAPE group: Second-look surgery plus hyper-thermic intraperitoneal chemotherapy versus surveillance in patients at high risk of developing colorectal peritoneal metas-tases (PROPHYLOCHIP-PRODIGE 15): A randomised, phase 3 study. Lancet Oncol. 2020, 21, 1147-1154. [CrossRef]

3. Klaver, C.E.L.; Wisselink, D.D.; Punt, C.J.A.; Snaebjornsson, P.; Crezee, J.; Aalbers, A.G.J. COLOPEC collaborators group: Adjuvant hyperthermic intraperitoneal chemotherapy in patients with locally advanced colon cancer (COLOPEC): A multicentre, open-label, randomised trial. Lancet Gastroenterol. Hepatol. 2019, 4, 761-770. [CrossRef]

4. Ceelen, W. HIPEC with oxaliplatin for colorectal peritoneal metastasis: The end of the road? Eur. J. Surg. Oncol. (EJSO) 2019, 45, 400-402. [CrossRef] [PubMed]

5. Tanis, P.J.; Tuynman, J.B.; De Hingh, I.H.J.T. Results from the PROPHYLOCHIP-PRODIGE 15 trial. Lancet Oncol. 2020, 21 , e496. [CrossRef]

6. Zhang, X.; Wu, Q.; Wei, M.; Deng, X.; Gu, C.; Wang, Z. Oxaliplatin versus mitomycin C in HIPEC for peritoneal metastasis from colorectal cancer: A systematic review and meta-analysis of comparative studies. Int. J. Color. Dis. 2020, 35, 1831-1839. [CrossRef]

7. Horvath, P.; Beckert, S.; Königsrainer, A.; Nadalin, S.; Königsrainer, I. Cytoreductive surgery and hyperthermic intraperitoneal chemotherapy combined with liver resection for concurrent peritoneal and hepatic metastases of gastrointestinal and gynecological primary tumors. J. Visc. Surg. 2019, 156, 475-484. [CrossRef] [PubMed]

8. Goéré, D.; Souadka, A.; Faron, M.; Cloutier, A.S.; Viana, B.; Honoré, C.; Dumont, F.; Elias, D. Extent of Colorectal Peritoneal Carcinomatosis: Attempt to Define a Threshold Above Which HIPEC Does Not Offer Survival Benefit: A Comparative Study. Ann. Surg. Oncol. 2015, 22, 2958-2964. [CrossRef]

9. Glehen, O.; Gilly, F.N.; Arvieux, C.; Cotte, E.; Boutitie, F.; Mansvelt, B.; Bereder, J.M.; Lorimier, G.; Quenet, F.; Elias, D. Peritoneal carci-nomatosis from gastric cancer: A multi-institutional study of 159 patients treated by cytoreductive surgery combined with perioperative intraperitoneal chemotherapy. Ann. Surg. Oncol. 2010, 17, 2370-2377. [CrossRef]

10. Zanon, C.; Clara, R.; Chiappino, I.; Bortolini, M.; Cornaglia, S.; Simone, P.; Bruno, F.; De Riu, L.; Airoldi, M.; Pedani, F. Cytoreductive Surgery and Intraperitoneal Chemohyperthermia for Recurrent Peritoneal Carcinomatosis from Ovarian Cancer. World J. Surg. 2004, 28, 1040-1045. [CrossRef]

11. Di Giorgio, A.; Naticchioni, E.; Biacchi, D.; Sibio, S.; Accarpio, F.; Rocco, M.; Tarquini, S.; Di Seri, M.; Ciardi, A.; Montruccoli, D.; et al. Cytoreductive surgery (peritonectomy procedures) combined with hyperthermic intraperitoneal chemotherapy (HIPEC) in the treatment of diffuse peritoneal carcinomatosis from ovarian cancer. Cancer 2008, 113, 315-325. [CrossRef] [PubMed]

12. Cotte, E.; Glehen, O.; Mohamed, F.; Lamy, F.; Falandry, C.; Golfier, F.; Gilly, F.N. Cytoreductive surgery and intraperitoneal chemo-hyperthermia for chemo-resistant and recurrent advanced epithelial ovarian cancer: Prospective study of 81 patients. World J. Surg. 2007, 31, 1813-1820. [CrossRef]

13. Van Oudheusden, T.R.; Braam, H.J.; Nienhuijs, S.W.; Wiezer, M.J.; van Ramshorst, B.; Luyer, P.; de Hingh, I.H. Poor outcome after cy-toreductive surgery and HIPEC for colorectal peritoneal carcinomatosis with signet ring cell histology. J. Surg. Oncol. 2015, 111, 237-242. [CrossRef]

14. Solomon, D.; DeNicola, N.; Ba, D.F.; Liu, P.H.; Pa, S.A.; Golas, B.J.; Sarpel, U.; Labow, D.M.; Magge, D.R. Signet ring cell features with peritoneal carcinomatosis in patients undergoing cytoreductive surgery and hyperthermic intraperitoneal chemotherapy are associated with poor overall survival. J. Surg. Oncol. 2019, 119, 758-765. [CrossRef]

15. Überrück, L.; Nadiradze, G.; Yurttas, C.; Königsrainer, A.; Königsrainer, I.; Horvath, P. In-Hospital Mortality and Complication Rates According to Health Insurance Data in Patients Undergoing Hyperthermic Intraperitoneal Chemotherapy for Peritoneal Surface Malignancies in Germany. Ann. Surg. Oncol. 2020, 1-8. [CrossRef]

16. Elias, D.; Gilly, F.; Boutitie, F.; Quenet, F.; Bereder, J.M.; Mansvelt, B.; Lorimier, G.; Dubè, P.; Glehen, O. Peritoneal colorectal carcino-matosis treated with surgery and perioperative intraperitoneal chemotherapy: Retrospective analysis of 523 patients from a multicentric French study. J. Clin. Oncol. 2010, 28, 63-68. [CrossRef]

17. Dindo, D.; Demartines, N.; Clavien, P.A. Classification of surgical complications: A new proposal with evaluation in a cohort of 6336 patients and results of a survey. Ann. Surg. 2004, 240, 205-213. [CrossRef] [PubMed]

18. Jacquet, P.; Sugarbaker, P.H. Clinical research methodologies in diagnosis and staging of patients with peritoneal carcinomato-sis. Cancer Treat. Res. 1996, 82, 359-374.

19. Spratt, J.S.; Adcock, R.A.; Muskovin, M.; Sherrill, W.; McKeown, J. Clinical delivery system for intraperitoneal hyperthermic chem-otherapy. Cancer Res. 1980, 40, 256-260. 
20. Kusamura, S.; Baratti, D.; Deraco, M. Multidimensional analysis of the learning curve for cytoreductive surgery and hyperther-mic intraperitoneal chemotherapy in peritoneal surface malignancies. Ann. Surg. 2012, 255, 348-356. [CrossRef] [PubMed]

21. Huang, Y.; Alzahrani, N.A.; Liauw, W.; Morris, D.L. Learning curve for cytoreductive surgery and perioperative intraperitoneal chemotherapy for peritoneal carcinomatosis. ANZ J. Surg. 2017, 87, 49-54. [CrossRef]

22. Hentzen, J.E.K.R.; Constansia, R.D.N.; Been, L.B.; Hoogwater, F.J.H.; Van Ginkel, R.J.; Van Dam, G.M.; Hemmer, P.H.J.; Kruijff, S. Diagnostic Laparoscopy as a Selection Tool for Patients with Colorectal Peritoneal Metastases to Prevent a Non-therapeutic Laparotomy During Cytoreductive Surgery. Ann. Surg. Oncol. 2019, 27, 1084-1093. [CrossRef]

23. Iversen, L.H.; Rasmussen, P.C.; Laurberg, S. Value of laparoscopy before cytoreductive surgery and hyperthermic intraperitoneal chemotherapy for peritoneal carcinomatosis. BJS 2012, 100, 285-292. [CrossRef]

24. Glehen, O.; Gilly, F.N.; Boutitie, F.; Bereder, J.M.; Quenet, F.; Sideris, L.; Mansvelt, B.; Lorimier, G.; Msika, S.; Elias, D. Toward curative treatment of peritoneal carcinomatosis from nonovarian origin by cytoreductive surgery combined with perioperative intra-peritoneal chemotherapy: A multi-institutional study of 1290 patients. Cancer 2010, 116, 5608-5618. [CrossRef] [PubMed]

25. Baratti, D.; Kusamura, S.; Mingrone, E.; Balestra, M.R.; Laterza, B.; Deraco, M. Identification of a Subgroup of Patients at Highest Risk for Complications after Surgical Cytoreduction and Hyperthermic Intraperitoneal Chemotherapy. Ann. Surg. 2012, 256, 334-341. [CrossRef]

26. Chua, T.C.; Moran, B.J.; Sugarbaker, P.H.; Levine, E.A.; Glehen, O.; Gilly, F.N.; Baratti, D.; Deraco, M.; Elias, D.; Sardi, A.; et al. Early- and Long-Term Outcome Data of Patients With Pseudomyxoma Peritonei From Appendiceal Origin Treated by a Strategy of Cytoreductive Surgery and Hyperthermic Intraperitoneal Chemotherapy. J. Clin. Oncol. 2012, 30, 2449-2456. [CrossRef]

27. Bakrin, N.; Bereder, J.; Decullier, E.; Classe, J.; Msika, S.; Lorimier, G.; Abboud, K.; Meeus, P.; Ferron, G.; Quenet, F.; et al. Peritoneal carcinomatosis treated with cytoreductive surgery and Hyperthermic Intraperitoneal Chemotherapy (HIPEC) for advanced ovarian carcinoma: A French multicentre retrospective cohort study of 566 patients. Eur. J. Surg. Oncol. (EJSO) 2013, 39, 1435-1443. [CrossRef]

28. Macrì, A.; Arcoraci, V.; Belgrano, V.; Caldana, M.; Cioppa, T.; Costantini, B.; Cucinotta, E.; De Cian, F.; De Iaco, P.; De Manzoni, G.; et al. Short-term outcome of cytoreductive surgery and hyperthermic intraperitoneal chemotherapy: Preliminary analysis of a multicentre study. Anticancer. Res. 2014, 34, 5689-5693. [PubMed]

29. Saxena, A.; Yan, T.D.; Chua, T.C.; Morris, D.L. Critical Assessment of Risk Factors for Complications After Cytoreductive Surgery and Perioperative Intraperitoneal Chemotherapy for Pseudomyxoma Peritonei. Ann. Surg. Oncol. 2009, 17, 1291-1301. [CrossRef]

30. Yang, X.-J.; Huang, C.-Q.; Suo, T.; Mei, L.-J.; Yang, G.-L.; Cheng, F.-L.; Zhou, Y.-F.; Xiong, B.; Yonemura, Y.; Li, Y. Cytoreductive Surgery and Hyperthermic Intraperitoneal Chemotherapy Improves Survival of Patients with Peritoneal Carcinomatosis from Gastric Cancer: Final Results of a Phase III Randomized Clinical Trial. Ann. Surg. Oncol. 2011, 18, 1575-1581. [CrossRef] [PubMed]

31. Bonnot, P.M.; Piessen, G.; Kepenekian, V.; Decullier, E.; Pocard, M.; Meunier, B.; Bereder, J.M.; Abboud, K.; Marchal, F.; Quenet, F.; et al. FREGAT and BIG-RENAPE Networks. Cytoreductive Surgery with or without Hyperther-mic Intraperitoneal Chemotherapy for Gastric Cancer with Peritoneal Metastases (CYTO-CHIP Study): A Propensity Score Analysis. J. Clin. Oncol. 2019, 37, 2028-2040. [CrossRef] [PubMed]

32. Witkamp, A.J.; de Bree, E.; Kaag, M.M.; Boot, H.; Beijnen, J.H.; van Slooten, G.W.; van Coevorden, F.; Zoetmulder, F.A. Extensive cy-toreductive surgery followed by intra-operative hyperthermic intraperitoneal chemotherapy with mitomycin-C in patients with peritoneal carcinomatosis of colorectal origin. Eur. J. Cancer 2001, 37, 979-984. [CrossRef]

33. Lambert, L.A.; Armstrong, T.S.; Lee, J.J.; Liu, S.; Katz, M.H.; Eng, C.; Wolff, R.A.; Tortorice, M.L.; Tansey, P.; Gonzalez-Moreno, S.; et al. Incidence, risk factors, and impact of severe neutropenia after hyperthermic intraperitoneal mitomycin C. Ann. Surg. Oncol. 2009, 16, 2181-2187. [CrossRef]

34. Adileh, M.; Mor, E.; Assaf, D.; Benvenisti, H.; Laks, S.; Ben-Yaacov, A.; Schtrechman, G.; Hazzan, D.; Shacham-Shmueli, E.; Margalit, O.; et al. Perioperative and Oncological Outcomes of Combined Hepatec-tomy with Complete Cytoreduction and Hyperthermic Intraperitoneal Chemotherapy for Metastatic Colorectal Cancer. Ann. Surg. Oncol. 2021, 28, 3320-3329. [CrossRef]

35. Hall, B.; Padussis, J.; Foster, J.M. Cytoreduction and Hyperthermic Intraperitoneal Chemotherapy in the Management of Colo-rectal Peritoneal Metastasis. Surg. Clin. N. Am. 2017, 97, 671-682. [CrossRef]

36. Franko, J.; Shi, Q.; Meyers, J.P.; Maughan, T.S.; Adams, R.A.; Seymour, M.T.; Saltz, L.; Punt, C.J.A.; Koopman, M.; Tournigand, C.; et al. Prognosis of patients with peritoneal metastatic colorectal cancer given systemic therapy: An analysis of individual patient data from prospective randomised trials from the Analysis and Research in Cancers of the Digestive System (ARCAD) database. Lancet Oncol. 2016, 17, 1709-1719. [CrossRef]

37. Schneider, M.A.; Eden, J.; Pache, B.; Laminger, F.; Lopez-Lopez, V.; Steffen, T.; Hübner, M.; Kober, F.; Roka, S.; Campos, P.C.; et al. Mutations of RAS/RAF Pro-to-oncogenes Impair Survival After Cytoreductive Surgery and HIPEC for Peritoneal Metastasis of Colorectal Origin. Ann Surg. 2018, 268, 845-853. [CrossRef]

38. Arjona-Sanchez, A.; Rodriguez-Ortiz, L.; Baratti, D.; Schneider, M.A.; Gutiérrez-Calvo, A.; García-Fadrique, A.; Tuynman, J.B.; Cascales-Campos, P.A.; Martín, V.C.; Morales, R.; et al. RAS Mutation Decreases Overall Sur-vival After Optimal Cytoreductive Surgery and Hyperthermic Intraperitoneal Chemotherapy of Colorectal Peritoneal Me-tastasis: A Modification Proposal of the Peritoneal Surface Disease Severity Score. Ann. Surg. Oncol. 2019, 26, 2595-2604. [CrossRef] 
39. Morgan, Z.; Chow, B.E.; Strong, E.A.; Tsai, S.; Christians, K.; Mogal, H.; Gamblin, T.C.; Clarke, C.N. RAS Mutation Status Confers Prognostic Relevance in Patients Treated With Cytoreductive Surgery and Hyperthermic Intraperitoneal Chemotherapy for Colorectal Cancer. J. Surg. Res. 2019, 240, 130-135. [CrossRef]

40. Baratti, D.; Kusamura, S.; Niger, M.; Perrone, F.; Milione, M.; Cattaneo, L.; Guaglio, M.; Bartolini, V.; Pietrantonio, F.; Deraco, M. Prog-nostic Impact of Primary Side and RAS/RAF Mutations in a Surgical Series of Colorectal Cancer with Peritoneal Metastases. Ann. Surg. Oncol. 2021, 28, 3332-3342. [CrossRef]

41. Graf, W.; Cashin, P.H.; Ghanipour, L.; Enblad, M.; Botling, J.; Terman, A.; Birgisson, H. Prognostic Impact of BRAF and KRAS Muta-tion in Patients with Colorectal and Appendiceal Peritoneal Metastases Scheduled for CRS and HIPEC. Ann. Surg. Oncol. 2020, 27, 293-300. [CrossRef]

42. Rau, B.; Brandl, A.; Piso, P.; Pelz, J.; Busch, P.; Demtröder, C.; Schüle, S.; Schlitt, H.J.; Roitman, M.; Tepel, J.; et al. Peritoneum Surface Oncology Group and members of the StuDoQ I Peritoneum Registry of the German Society for General and Visceral Surgery (DGAV). Peritoneal metastasis in gastric cancer: Results from the German database. Gastric Cancer 2020, 23 , 11-22. [CrossRef]

43. Spiliotis, J.; Halkia, E.; Lianos, E.; Kalantzi, N.; Grivas, A.; Efstathiou, E.; Giassas, S. Cytoreductive Surgery and HIPEC in Recurrent Epithelial Ovarian Cancer: A Prospective Randomized Phase III Study. Ann. Surg. Oncol. 2015, 22, 1570-1575. [CrossRef] [PubMed]

44. Fagotti, A.; Costantini, B.; Petrillo, M.; Vizzielli, G.; Fanfani, F.; Margariti, P.A.; Turco, L.C.; Piovano, E.; Scambia, G. Cytoreductive sur-gery plus HIPEC in platinum-sensitive recurrent ovarian cancer patients: A case-control study on survival in patients with two year follow-up. Gynecol. Oncol. 2012, 127, 502-505. [CrossRef]

45. Van Driel, W.J.; Koole, S.N.; Sikorska, K.; Schagen van Leeuwen, J.H.; Schreuder, H.W.R.; Hermans, R.H.M.; de Hingh, I.H.J.T.; van der Velden, J.; Arts, H.J.; Massuger, L.F.A.G.; et al. Hyperthermic Intraperitoneal Chemotherapy in Ovarian Cancer. N. Engl. J. Med. 2018, 378, 230-240. [CrossRef] [PubMed]

46. Coleman, R.L.; Spirtos, N.M.; Enserro, D.; Herzog, T.J.; Sabbatini, P.; Armstrong, D.K.; Kim, J.-W.; Park, S.-Y.; Kim, B.-G.; Nam, J.-H.; et al. Secondary Surgical Cytoreduction for Recurrent Ovarian Cancer. N. Engl. J. Med. 2019, 381, 1929-1939. [CrossRef]

47. Wang, Y.; Ren, F.; Chen, P.; Liu, S.; Song, Z.; Ma, X. Effects of CytoReductive surgery plus hyperthermic IntraPEritoneal chemotherapy (HIPEC) versus CytoReductive surgery for ovarian cancer patients: A systematic review and meta-analysis. Eur. J. Surg. Oncol. 2019, 45, 301-309. [CrossRef]

48. Wu, Q.; Wu, Q.; Xu, J.; Cheng, X.; Wang, X.; Lu, W.; Li, X. Efficacy of hyperthermic intraperitoneal chemotherapy in patients with epithelial ovarian cancer: A meta-analysis. Int. J. Hyperth. 2019, 36, 561-571. [CrossRef] [PubMed]

49. Kim, S.I.; Cho, J.; Lee, E.J.; Park, S.; Park, S.J.; Seol, A.; Lee, N.; Yim, G.W.; Lee, M.; Lim, W.; et al. Selection of patients with ovarian cancer who may show survival benefit from hyperthermic intraperitoneal chemotherapy: A system-atic review and meta-analysis. Medicine 2019, 98, e18355. [CrossRef]

50. Cianci, S.; Riemma, G.; Ronsini, C.; De Franciscis, P.; Torella, M.; Schiattarella, A.; La Verde, M.; Colacurci, N. Hyperthermic intraper-itoneal chemotherapy (HIPEC) for ovarian cancer recurrence: Systematic review and meta-analysis. Gland Surg. 2020, 9 , 1140-1148. [CrossRef] 